\title{
DISPOSITIF DE PULSATION DU FAISCEAU D'UN ACCÉLÉRATEUR VAN DE GRAAFF DE 3 MEV
}

\author{
Par G. BOUGNOT, R. GALIANA, J. SAMUELI et A. SARAZIN, \\ Institut d'Études Nucléaires d'Alger.
}

\begin{abstract}
Résumé. - On décrit un dispositif électrostatique permettant l'obtention d'impulsions de protons inférieures à la nanoseconde avec une fréquence de répétition de $10 \mathrm{MHz}$. Le courant moyen obtenu est de $0,1 \mu \mathrm{A}$. Les circuits électroniques de chronométrage associés au dispositif de pulsation sont également décrits.
\end{abstract}

Abstract. - We describe an electrostatic apparatus allowing to obtain proton pulses, the duration of which are less than a nanosecond, with a repetitive rate of $10 \mathrm{MHz}$.

The average current reached is about $0.1 \mu \mathrm{A}$.

The time measurement electronic circuits related to the pulse system are also described.

Introduction. - Le principe de l'obtention d'impulsions périodiques de particules par déflexion électrostatique du faisceau est bien connu $[1,2,3]$ : on dirige le faisceau entre des plaques sur lesquelles est appliquée une tension sinusoïdale de telle façon qu'il soit dévié alternativement vers chacune de ces plaques. Il suffit de placer un diaphragme percé d'un orifice pour produire une impulsion de particules à chaque passage du faisceau. On obtient ainsi des impulsions d'autant plus courtes que la vitesse de balayage est grande et que l'orifice du diaphragme est petit. En fait, avec un tel dispositif on obtient deux impulsions de particules par période du signal sinusoïdal ce qui peut créer une difficulté de repérage dans le temps. En effet, si le diaphragme de pulsation n'est pas exactement positionné au centre du segment de droite décrit par le faisceau, les deux impulsions ne sont pas séparées d'une demi période mais de deux valeurs successives différentes. Pour éviter cette difficulté, nous avons adopté un balayage en ellipse obtenu par deux ensembles de déflexion, l'un horizontal, l'autre vertical recevant des tensions convenablement déphasées.

Montage expérimental. - La figure 1 représente le montage. L'accélérateur Van de Graaff de $3 \mathrm{MeV}$ est disposé verticalement. L'aimant analyseur introduit une déflection de $90^{\circ}$. Après les fentes de stabilisation entre lesquelles est focalisé le fajsceau, nous avons disposé un diaphragme de $3 \mathrm{~mm}$ de diamètre destiné à limiter sa divergence avant l'entrée dans une paire de lentilles quadrupolaires électrostatiques (fig. 2). Ces lentilles refocalisent le faisceau sur le diaphragme de pulsation. Nous obtenons ainsi.un courant de protons compris entre 5

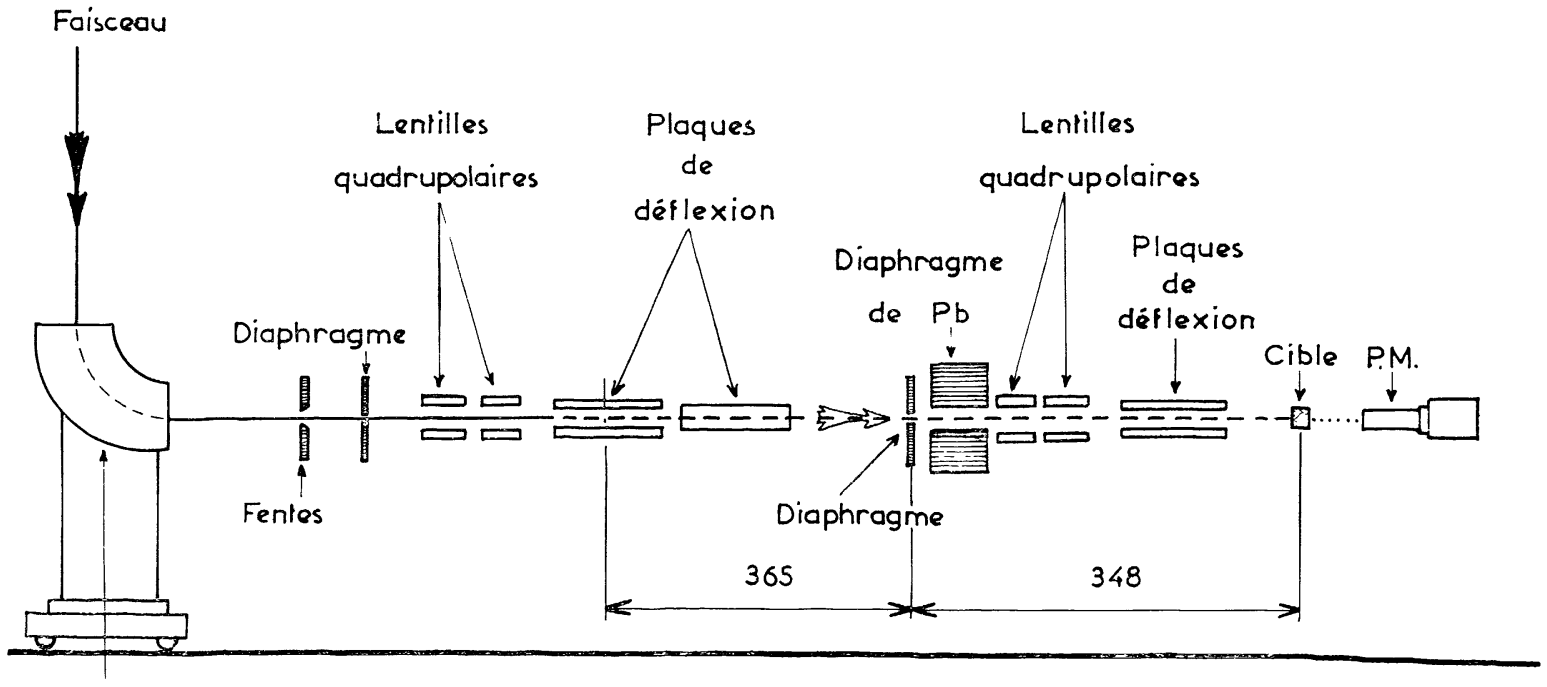

Eleciro-aimant

FIG. 1. 

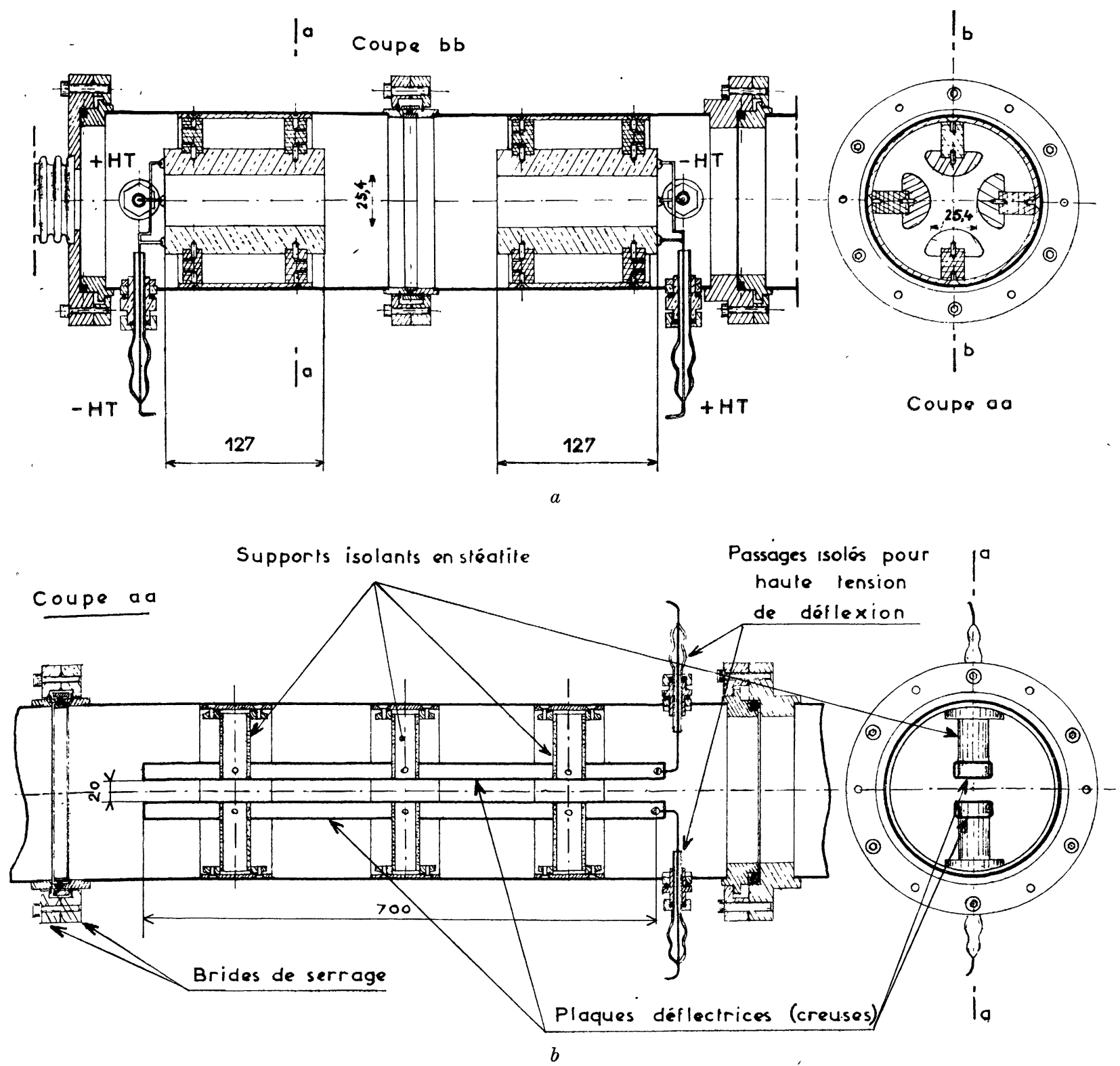

FIG. 2,

et $10 \mu \mathrm{A}$ à l'entrée des lentilles qui sont placées immédiatement avant les premières plaques déflectrices disposées horizontalement. Lo diaphrag ne de pulsation est constitué d'une plaque méta!lique percée d'un orifice de $3 \mathrm{~mm}$ et recouverte d'une plaque de verre métallisé qui permet à l'aide d'un miroir à $45^{\circ}$ et d'une lucarne, d'observer l'e'lipse décrite par le faisceau. Los secondes plaques défl эctrices sont disposées verticalement, une tension continue appliquée sur l'une de ces plaques déplace l'ellipse dans son ensemble pour qu'une de ses branches passe sur l'orifice du diaphragme. Le bloc de plomb placé à proximité arrête une partie des rayonnements produits sur le diaphragme. Pour éviter ce rayonnement parasite la cible est éloignée d'une certains distance. Une seconde paire de lentilles électrostatiques permet la refocalisation sur la cible.

Une paire de plaques déflectrices supplémentaire est disposée après ces dernières lentilles. Elle permet l'utilisation de plusieurs cibles en bout de montage, une polarisation continue appliquée sur les plaques permettant de défléchir le faisceau pour atteindre la cible choisie. Ce dispositif inspiré de celui décrit par Birk [3] permet d'effectuer successivement des mesures sur diverses cibles et 


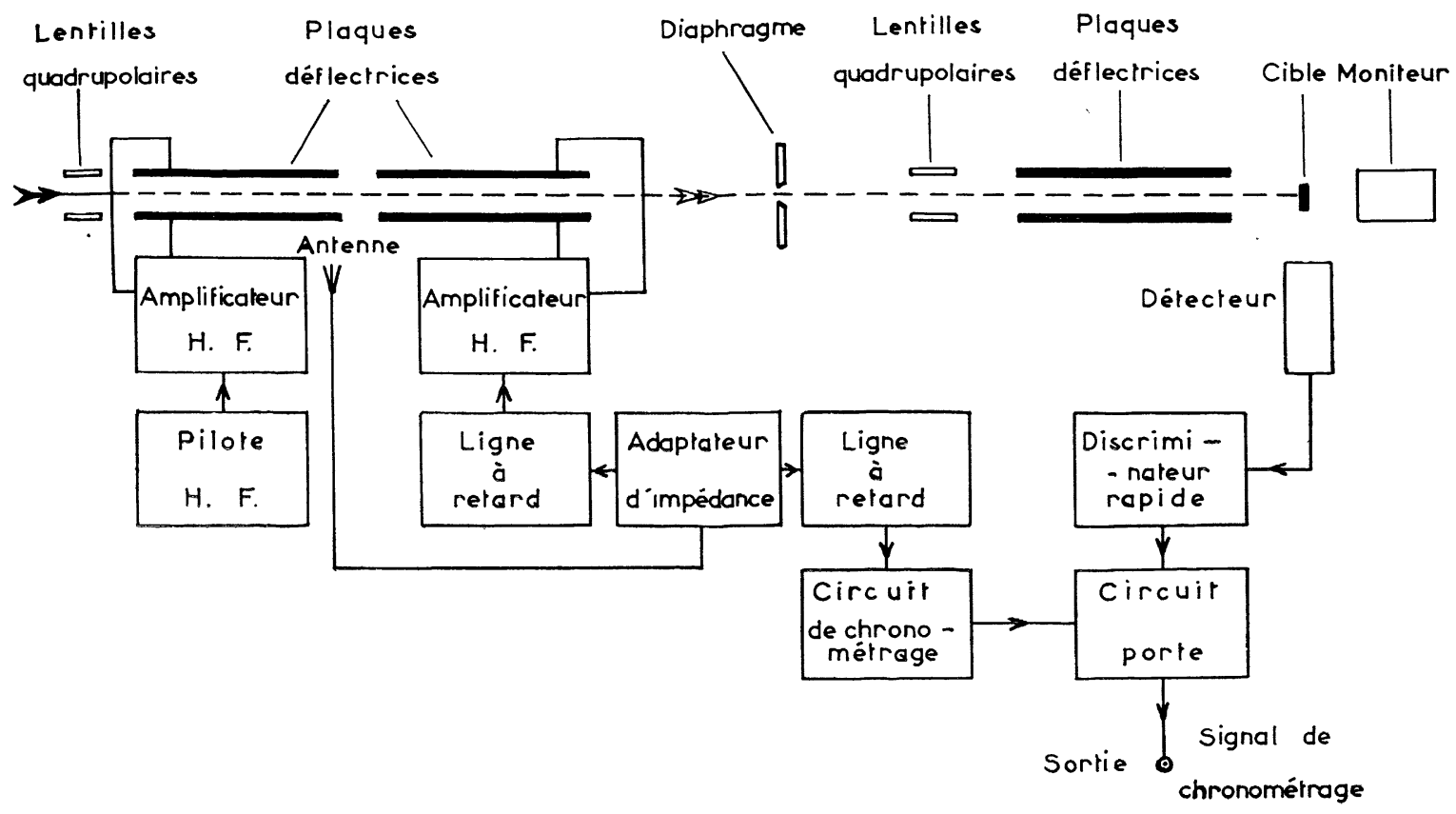

FIG. 3.

de déterminer, par exemple, l'origine des temps en utilisant des noyaux dont la durée de vie du premier niveau excité est très brève.

Circuits électroniques. - La figure 3 représente le montage électronique du dispositif de pulsation et de chrono métrage.

Pulsation. - Deux circuits amplificateurs sont utilisés pour fournir la puissance haute fréquence aux plaques déflectrices. Le pilotage est effectué par quartz. Chacun des circuits d'alimentation des déflecteurs est constitué d'un préamplificateur (EL 84 + 2X6 L 6) suivi d'un étage de puissance (P 600A CSF) fonctionnant en classe C. La charge anodique de ce tube est un circuit oscillant dont les plaques déflectrices constituent la capacité. Ces plaques ( $f i g .2$ ) ont une longueur de $70 \mathrm{~cm}$, une largeur de 2,54 cm et sont distantes de $2 \mathrm{~cm}$. L'inductance anodique est connectée en autotransformateur de rapport 5,5 environ. Les alimentations haute tension des tubes $\mathrm{P} 600 \mathrm{~A}$ sont réglables entre 1000 et 3000 volts et permettent ainsi que les injections variables, le réglage des tensions haute fréquence. Pour éviter une dérive trop importante du déphasage entre les tensions haute fréquence appliquées, le second amplificateur est alimenté non par le pilote, mais par la tension recueillie aux bornes d'une boucle placée près du circuit oscillant du premier amplificateur.

Un adaptateur d'impédance et une ligne à retard réglable sont insérés dans ce circuit permettant un choix aisé du déphasage entre les deux déflecteurs.
La même boucle alimente le circuit de chrono métrage et un voltmètre donnant une indication relative de la tension aux bornes du premier déflecteur. La puissance maximum disponible aux bornes de chaque amplificateur est de l'ordre de 400 watts, la tension crête à crête aux bornes du déflecteur étant alors de 8000 volts environ.

Circuit de chronométrage. - Ce circuit a pour but de fournir un signal décalé d'un temps constant par rapport à l'instant d'émission d'une impulsion de protons. L'intensité du faisceau étant assez faible, la méthode la plus simple pour déterminer cet instant est de produire un signal dérivé des tensions sinusoïdales appliquées aux déflecteurs. Nous avons indiqué précédemment que la tension sinusoïdale provient d'une boucle couplée au circuit anodique final du premier amplificateur haute fréquence. Cette méthode d'alimentation a pour but d'éliminer les variations de déphasage pouvant intervenir dans l'amplificateur, le signal aux bornes de la boucle étant synchrone de celui appliqué aux plaques de déflexion. En fait, il est utile d'associer un circuit porte au circuit de chronométrage car le signal de chronométrage n'est utile que lorsqu'un événement nucléaire lié à l'arrivée de l'impulsion de protons sur la cible aura été détecté. La figure 4 indique le schéma de ces circuits. Le circuit de chronométrage est constitué des tubes $V_{1}$ à $\left[V_{5}\right.$. $\mathrm{V}_{1}$ est polarisé de façon à ne laisser passer que les alternances positives de la tension sinusoïdale d'entrée. Après amplification par $V_{2}$ et $V_{3}$ les signaux sont coupés par câble dans l'anode de $V_{4}$. 

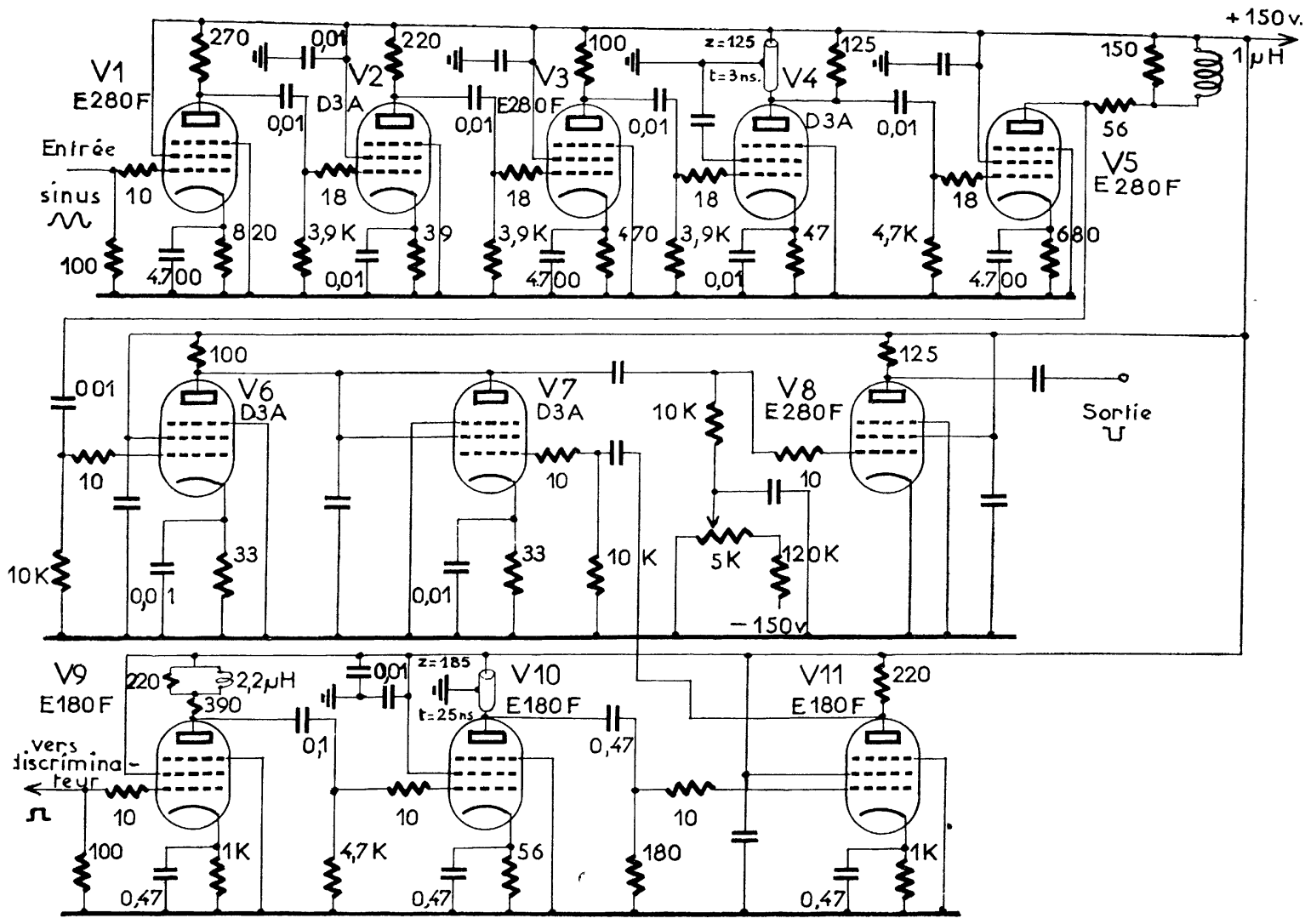

FIG. 4.

$\mathrm{V}_{\mathbf{5}}$ élimine les parties négatives des impulsions résultantes. On applique donc à la première entrée du circuit d'addition $\left(\mathrm{V}_{6}\right)$ un train de signaux négatifs d'amplitude 2 volts et de largeur $7 \mathrm{~ns}$ environ dont la fréquence de répétition est celle de la tension. Le circuit porte, quant à lui, ne doit laisser passer un signal de chronométrage qu'en présence d'un signal d'ouverture.

Les tubes $V_{\mathbf{9}}, V_{10}$ et $V_{11}$ transforment ce signal en impulsion rectangulaire de $50 \mathrm{~ns}$ de large et d'amplitude 2 volts qui est envoyée sur la grille de $V_{7}$. La fonction porte est réalisée par le circuit d'addition $\left(V_{6}\right.$ et $\left.V_{7}\right)$ et par le tube $V_{8}$ qui effectue une discrimination à seuil légèrement supérieur à l'amplitude commune des deux signaux additionnés. Après discrimination, seul sort le signal de chronométrage arrivé pendant le temps d'ouverture de 50 ns.

\section{BIBLIOGRAPHIE}

[1] Good (W. M.), Nucl. Inst., 1960, 6, 323.

[2] Ballini (R.) et Kuo (L. G.), J. Physique Rad., 1959, $20,47 \mathrm{~A}$
[3] Birk (M.), Goldring (G.) et Wolfson (Y.), Phys. Res., 1959, 116, 730 . 